\title{
Civilisations
}

Revue internationale d'anthropologie et de sciences

humaines

$41 \mid 1993$

Mélanges Pierre Salmon II

\section{L'exploitation du caoutchouc par l'état indépendant du Congo dans le territoire de Banzyville, district de l'Ubangi (1900-1908)}

Te Mobusa Ngbwapkwa

\section{(2) OpenEdition}

\section{Journals}

Édition électronique

URL : http://journals.openedition.org/civilisations/1713

DOI : 10.4000/civilisations. 1713

ISSN : 2032-0442

Éditeur

Institut de sociologie de l'Université Libre de Bruxelles

Édition imprimée

Date de publication : 1 septembre 1993

Pagination : 291-306

ISBN : 2-87263-094-5

ISSN : 0009-8140

Référence électronique

Te Mobusa Ngbwapkwa, « L'exploitation du caoutchouc par l'état indépendant du Congo dans le territoire de Banzyville, district de l'Ubangi (1900-1908) », Civilisations [En ligne], 41 | 1993, mis en ligne le 30 juillet 2009, consulté le 21 décembre 2020. URL : http://journals.openedition.org/civilisations/ 1713 ; DOI : https://doi.org/10.4000/civilisations. 1713

Ce document a été généré automatiquement le 21 décembre 2020.

(c) Tous droits réservés 


\title{
L'exploitation du caoutchouc par l'état indépendant du Congo dans le territoire de Banzyville, district de l'Ubangi (1900-1908)
}

\author{
Te Mobusa Ngbwapkwa
}

\section{Introduction}

1 Confronté à des difficultés financières et convaincu de l'idée qu'une colonie doit être rentable pour sa mère-patrie ${ }^{1}$, Léopold II prend, à partir de 1892, une série de mesures destinées à lui procurer de l'argent, c'est-à-dire à rentabiliser le Congo. Certaines régions sont déclarées "domaine privé" de l'Etat. Les bassins de l'Ubangi, du Mbomu et de l'Uele, les bassins de la Mongala, de l'Itimbiri et de l'Aruwimi, les bassins des rivières Lopori et Maringa ainsi que les régions de Busira et la Tchwapa sont constitués en domaine privé2. L'exploitation du caoutchouc par des particuliers dans ce domaine privé n'est pas autorisée. Une partie des territoires précités sera cependant concédée aux sociétés "privées". La Société Anversoise pour le Commerce au Congo ("l'Anversoise") recevra le bassin de la Mongala, tandis que l'Anglo-Belgium India Rubber ("l'Abir") aura le bassin de la Lopori-Maringa ${ }^{3}$.

2 En 1896, les bassins de la Busira et de la Momboyo, les bassins du lac Léopold II et de la Lukenie sont érigés en Domaine de la Couronne ${ }^{4}$. Domaine privé ou domaine de la Couronne, l'exploitation du caoutchouc dans ces territoires est faite par les agents de l'Etat Indépendant du Congo.

3 Les témoignages des missionnaires protestants américains, suédois et anglais, le rapport du consul britannique Roger Casement et de nombreux articles de la Congo Reform Association de E.D. Morel ont révélé les conditions de travail très dures et quasi inhumaines des populations dans le Domaine de la Couronne. Ils ont révélé également 
que des atrocités avaient été commises par les agents de l'Etat ${ }^{5}$. Cependant on ne s'est jamais fait une idée claire sur les conditions de travail des populations vivant dans un territoire comme Banzyville. Notons que ce dernier territoire, bien que ne faisant pas directement partie du Domaine de la Couronne, était malgré tout exploité directement par l'Etat.

Notre étude se propose d'apporter une modeste contribution destinée à éclairer cette page d'histoire de l'Etat Indépendant du Congo dans le territoire de Banzyville. Elle se base sur les rapports politiques totalement inédits de 1902 à 1908 . Ce document est conservé dans les Archives des Pères Capucins à Anvers. L'étude sera axée sur deux points : l'exploitation du caoutchouc et les conséquences qui en ont résulté.

\section{L'exploitation du caoutchouc dans le territoire de l'Ubangi}

5 L'exploitation du caoutchouc dans le territoire de Banzyville a débuté assez tardivement, en $1900^{6}$. Ce retard s'explique surtout par le manque de personnel pouvant se consacrer à ce travail. En avril 1896, le lieutenant Louis Royaux ${ }^{7}$ est désigné pour le district de l'Ubangi et est chargé de l'exploitation du caoutchouc dans ce territoire. Arrivé à Boma le 25 octobre, il doit, sur ordre du Gouverneur Général, effectuer un stage de deux semaines dans le district de l'Equateur, sous les ordres du commissaire Fiévez ${ }^{8}$. Ce dernier doit l'initier aux procédés de la récolte du caoutchouc, mais aussi aux méthodes pouvant permettre un accroissement rapide de la production.

6 Victor Fiévez était surnommé "Ntange", c'est-à-dire "lit" par les populations, parce qu'il adorait faire la sieste à midi ${ }^{9}$. Malgré ce surnom tranquille, Fiévez avait une très mauvaise réputation dans la région. Il est l'une des principales figures accusées d'atrocités congolaises en 1895 et en $1896^{10}$. On peut se demander quelle méthode il pouvait enseigner à son jeune stagiaire. Louis Royaux arrive dans le district de l'Ubangi en avril 1897 ; il est chargé de réoccuper le poste d'Imesse dans le Sud-Ubangi, poste abandonné depuis 1896 à cause de l'hostilité des populations aux impositions du caoutchouc. Il est également chargé de bâtir à l'emplacement de l'ancien village Libenge une station de l'Etat destinée à devenir le chef lieu du district ${ }^{11}$.

7 C'est en décembre 1898 que Royaux arrive dans le territoire de Banzyville, mais il ne peut commencer la récolte du caoutchouc car, sur ordre impératif du Gouverneur Général, il doit recruter le plus grand nombre d'hommes possible et les diriger vers le nord (Uele) pour lutter contre les Batetela révoltés depuis $1897^{12}$. C'est seulement en 1900 que Louis Royaux peut faire débuter sa principale tâche, la récolte du caoutchouc.

8 Tous les villages dépendant du poste de Banzyville ont reçu chacun un rôle : récolteur, pagayeur, ravitailleur, confectionneur des paniers à caoutchouc... Sont chargés de la récolte du caoutchouc les villages suivants: à l'est de Banzyville, Basa, chef Yendani, Vote, chef Deba, Dondo, chef Kenga et Ndekere, chef Kutene. A l'ouest de Banzyville, on signale les villages Ngonda, chef Mbaya, village Lite de Gbado, chef Kaya, Lite de Molegbe, chef Gbwadembi ; Bakpa, chef Guyombo et quelques villages Mbanza. Les Gbanziri et les Saka, en aval, les Ndia en amont de Banzyville et les Sango, les Kumbu, les Ziamba et les Mune, autour du poste, sont chargés de transport en pirogues du caoutchouc vers Mokwangai, Zongo et Libenge. Les Monge sont chargés de confectionner les paniers à caoutchouc tandis que les Nzakara doivent ravitailler le poste en vivres frais ${ }^{13}$. 
L'année 1900 est consacrée à l'enseignement à chaque village des procédés de la récolte du caoutchouc. Les Ngbandi, qui sont déjà au courant du travail du caoutchouc, ont essayé, mais en vain, de persuader Louis Royaux de l'inexistence de lianes et arbres à caoutchouc dans leurs forêts. "Tous mes braves gens, écrit Royaux, n'ignorent point les conséquences futures de cette nouvelle décision de Boula Matari. L'écho trop souvent tragique de ce qui se passe dans la Mongala n'est pas pour les rassurer" ${ }^{14}$.

Le lieutenant Louis Royaux quitte Banzyville le premier février 1901. Il y est remplacé par le lieutenant Rodolphe Arnold ${ }^{15}$. Ce dernier a une réputation semblable à celle de Léon Fiévez, commissaire du district de l'Equateur. Le lieutenant Arnold était chef de poste d'Ekuta, dans le Sud- Ubangi, où il était chargé de la récolte du caoutchouc. En janvier 1901, il avait attaqué le village Kanda (Mbanza) qui, disait-il, avait refusé de récolter du caoutchouc. Deux cents personnes avaient été tuées. Cette opération lui avait valu un simple blâme ${ }^{16}$. Sa mutation pour le poste de Banzyville était la sanction suprême. Dans le poste de Banzyville, Rodolphe Arnold est de nouveau chargé de la récolte du caoutchouc. Il laisse des souvenirs très amers dans la région. Durant une période de deux ans et dix mois qu'il a passé dans le territoire, Arnold s'est occupé de la récolte du caoutchouc avec des méthodes inhumaines. Il a été surnommé "Zebayi", c'est-à-dire le léopard de Mobayi. Ce surnom traduit la manière dont le Lieutenant Arnold traquait dans la forêt les réfractaires aux impositions ${ }^{17}$.

11 Rodolphe Arnold est remplacé en août 1903 par un Italien, le capitaine Giovanni Aiuti ${ }^{18}$, après une courte période intérimaire du capitaine Jacques Saubert ${ }^{19}$. Le capitaine Aiuti s'occupe comme Arnold de la récolte du caoutchouc jusqu'en juin 1905 avec des moyens très durs. Il quitte le poste en juin 1905. Il y est remplacé par un autre Italien, le capitaine Biboloni. Celui-ci doit céder sa place au capitaine Dubreucq ${ }^{20}$, après treize mois de travail. Le capitaine Biboloni commande le territoire de septembre 1906 à juillet 1908, avant de céder à nouveau le commandement au capitaine Jacques Saubert durant les quelques mois qui précèdent l'annexion du Congo par la Belgique.

Chacun des officiers cités ci-dessus a essayé, en ce qui le concerne, d'obtenir des populations la plus grande production possible de caoutchouc. En effet, pour activer la production, des avantages étaient accordés aux officiers et agents de l'Etat (systèmes de primes de dix pour cent ou d'avancement de grades) ${ }^{21}$. Aux avantages matériels s'ajoutaient les injonctions de Bruxelles réclamant toujours du caoutchouc et davantage de caoutchouc. Les officiers et agents de l'Etat étaient invités à ne rien négliger pour procurer de grandes quantités de ce produit à l'Etat ${ }^{22}$. C'est l'engrenage du système léopoldien ${ }^{23}$. Les officiers et agents de l'Etat, motivés par les primes et excités par les ordres pressants de Bruxelles, se livrent alors à des brutalités sur les populations pour obtenir d'elles la plus grande production possible.

Le chiffre de la production du caoutchouc manque pour les deux premières années c'està-dire de 1900 à 1901 ; par contre, du $1^{\mathrm{er}}$ janvier 1902 au 31 décembre 1908, il a été récolté au poste de Banzyville 152.646 kilos de caoutchouc ${ }^{24}$, soit une moyenne de 21.806 kilos par an. Cette production, peut-on dire, est insignifiante par rapport à celle de la Mongala, qui atteignait par moment 60.000 kilos par mois ${ }^{25}$. Il faut noter que la situation du poste de Banzyville est particulière. Plus de la moitié du territoire est couverte par la savane; la forêt n'occupe qu'une étroite bande ne dépassant pas vingt kilomètres de profondeur au Sud-Ouest de Banzyville. C'est dans cette forêt que tous les récolteurs doivent se rendre. Il en résulte des difficultés énormes pour eux de satisfaire à ces impositions. 
14 En 1904, la forêt précitée est épuisée. En effet, comme le souligne R. Vaquier, la récolte provenait de caoutchouc d'herbes (rhizomes) et de lianes, plantes sauvages dont l'indigène, travaillant à son compte sans surveillance, obtenait le latex par coupe et arrachages abusifs, détruisant les plantes et obligeant à aller de plus en plus loin pour en trouver ${ }^{26}$. L'épuisement de la "forêt des Kawele" amène le commissaire du district, le commandant Bertrand, à décider l'envoi du chef Yendani et son groupe pour récolter du caoutchouc dans la forêt des Nzale. Ces derniers dépendent de l'administration de Yakoma; 19 récolteurs Basa sont massacrés ${ }^{27}$. Devant la révolte des Nzale, le commissaire de district dirige tous les récolteurs de Banzyville dans la seule et unique forêt qui renfermait encore des arbres et lianes à caoutchouc et qui se trouvait à l'extrême SudOuest du poste de Banzyville, entre les rivières Ngoko et Lua-Vindu. Cette forêt est peuplée de Mbanza, de Ngbaka et de Ngombe, ce qui entraîne également des guerres entre les Ngbandi et les groupes précités ${ }^{28}$.

A partir de la deuxième moitié de 1904, la forêt de Lua-Vindu qui n'était pas grande - elle était limitée au sud par le domaine de l'Anversoise - doit accueillir journellement une moyenne de 1.000 récolteurs, ce qui entraîne son épuisement total en 1907. Les imposés ne parvenaient plus à s'acquitter de leurs obligations et ils se plaignaient amèrement. En mai 1907, le capitaine Dubreucq décide de s'y rendre en personne pour vérifier si les plaintes des récolteurs sont fondées. Voici ce qu'il écrit: "Grâce aux indigènes qui m'accompagnaient, j'ai pu me rendre compte de l'impossibilité absolue où se trouvent mes récolteurs de fournir la totalité de leurs impositions. Les irehs sont devenus rares et tout ce qui subsiste encore sont épuisés par de trop nombreuses saignées" 29 .

L'organisation du travail était la suivante: un tiers de chaque village récolteur partait dans la forêt le $1^{\mathrm{er}}$ mars, le deuxième tiers suivait le $1^{\mathrm{er}}$ mai et le troisième partait le $1^{\mathrm{er}}$ juillet. Le tiers parti le $1^{\text {er }}$ mars rentrait fin avril ; celui parti le $1^{\text {er }}$ mai retournait fin juin. Le troisième tiers, partant le $1^{\mathrm{er}}$ juillet, rentrait fin août. Le premier tiers repartira le $1^{\mathrm{er}}$ septembre et ainsi de suite ${ }^{30}$. L'organisation était conçue de telle manière que chaque homme travaillait deux cent vingt quatre heures tous les six mois, c'est-à-dire environ trente six heures par mois. Ce qui était loin d'être le cas. En effet, le nombre très élevé de récolteurs (en moyenne 1.000 personnes) qui se rendaient dans la forêt de Kawele et dans celle de Lua-Vindu, peu étendues et moins riches en arbres et lianes à caoutchouc, faisait qu'un kilo de latex était obtenu au prix de plusieurs journées de travail. Catherine Coquery-Vidrovitch souligne à ce propos qu'en 1911, au Congo français, il fallait, pour faire un kilo de caoutchouc, trente-huit heures au moins, soit quatre journées de travail à un ouvrier habile et minutieux travaillant sans relâche sur des lianes riches et situées à proximité du village ${ }^{31}$. Dans le rapport précité, le capitaine Dubreucq fait remarquer au Commissaire de district que le lieu de la récolte du caoutchouc est très éloigné du poste de Banzyville. "En prenant Banzyville même comme point de départ, il faut dix jours à l'aller, dix jours au retour, et huit pour récolter 6 à 10 kilos de caoutchouc ${ }^{132}$. Les trentesix par mois ne correspondaient nullement à la réalité.

17 Lorsqu'un village ne parvient pas à satisfaire ses obligations ou comme l'écrit Arnold, lorsqu'il vient à "se relâcher" au point de vue de la fourniture du caoutchouc, de vivres ou d'autres prestations, on organise "une visite" d'un détachement de police pour le mettre à la raison. Les visites militaires, promenades militaires... sont très nombreuses du temps du lieutenant Arnold et du capitaine Aiuti. Il est difficile de les reprendre toutes ici. Nous en donnons quatre ou cinq en guise d'illustration des atrocités commises par des officiers de l'Etat Indépendant du Congo chargés de la récolte du caoutchouc ${ }^{33}$. 
18 En janvier 1901, les Ndekere et les Vote se révoltent; ils massacrent quatre soldats (sentinelles) de la Force Publique. En guise de représailles, on décide une opération militaire combinée des détachements de Banzyville commandés par Louis Royaux et de Yakoma conduits par Auguste Gérard. Les forces de l'Etat, comprenant 98 hommes, attaquent le 2 février les villages cités ci-dessus et les occupent pendant 6 semaines. Le chef Kutene des Ndekere est tué et les Vote, à quelques exceptions près, s'enfuient sur la rive droite de l'Ubangi, en territoire françaiis ${ }^{34}$.

19 L'année suivante, le lieutenant Arnold, qui a succédé au poste de Banzyville à Louis Royaux, revient chez les Vote et les Ndekere. A la tête de 40 soldats, il attaque ces populations qui, après quelques escarmouches, "durs combats" selon Arnold, se réfugient dans les collines de Kota-Koli. Zebayi (c'est ici que Arnold reçut son surnom) les y poursuit. "A la fin les vivres leur manquaient; quelques uns de leurs hommes furent tués et beaucoup fait prisonniers. Lorsque j'eus fait assez grand nombre de prisonniers, je partis avec eux, et j'allai camper à l'ancien village Yigbo, en attendant la reddition des chefs. De nombreuses patrouilles circulaient dans les plantations pour empêcher les indigènes de venir y chercher des vivres" ${ }^{135}$. Contraints par la faim et les souffrances, le 5 mars, le chef Vote vient se livrer en compagnie de quelques notables; quelques jours après, le chef Ndekere vient également faire sa soumission. Les Vote et les Ndekere sont sommés de payer leurs impositions et "les nombreux arriérés dus après un si long chômage ${ }^{136}$. Il y a eu par la suite d'autres expéditions punitives contre les Vote et les Ndekere. Ces derniers ne se sont jamais complètement soumis avant 1908.

Des expéditions militaires ont été également dirigées contre les récolteurs à l'ouest de Banzyville. Le 11, 12 et 13 juin 1902, le lieutenant Arnold attaque le village Lite de Gbado accusé d'un "grand laisser-aller" dans la récolte du caoutchouc. "Ils ne viennent au poste qu'avec une faible partie de leur imposition, et encore ne l'apportent-ils que quelques jours après la date qui leur est fixée". Le chef oko est accusé de se montrer indocile. "Imposé pour 8 paniers de 35 kilos, il en apporte un ou deux, et son caoutchouc est de mauvaise qualité et plein de matière étrangère". Lors de cette opération, écrit Arnold, plusieurs coups de feu ont été tirés ${ }^{37}$. Les autres chefs Lite, effrayés par les massacres, viennent faire leur soumission.

21 Le 29 janvier 1904, le capitaine Aiuti, à la tête d'une force comprenant 21 soldats, deux caporaux et 55 pistonniers attaque le village Gbandu, village Mbanda à l'ouest de Banzyville. On reproche aux habitants d'avoir, lors du défrichement de leurs champs, coupé des arbres à caoutchouc. L'opération fait de très nombreuses victimes, comme en témoigne le passage suivant de l'officier de la Force Publique lui-même. "Dans ma visite au village, je trouvais 30 morts. Il doit y avoir eu bien des blessés, les traces de sang relevées sur les sentiers étaient considérables. La porte d'entrée était barricadée comme celle d'en-bas, et près d'elles, mêmes trophées de crânes humains" ${ }^{138}$. L'opération avait fait au total près de 60 morts, sans compter des blessés.

Le 7 mars 1904, le commissaire de district Bertrand arrive à Banzyville. Il donne ordre au capitaine Aiuti de diriger une opération de représailles contre les Nzale qui avaient tué 19 récolteurs Basa. Le capitaine Aiuti quitte Banzyville le 13 mars avec 96 soldats pour une opération de guerre contre les Nzale. Plusieurs personnes sont tuées ${ }^{39}$.

23 Conjointement à des opérations militaires, des prises d'otages sont régulièrement organisées ${ }^{40}$. Les chefs et les notables sont détenus au poste dans des conditions souvent humiliantes. Il est démontré toutefois que la méthode n'était pas efficace. "L'arrestation 
des chefs et de notables ne les effraie pas. Les administrés ne font pas un gramme en plus pour les délivrer" ${ }^{141}$. Seules les opérations de guerre peuvent, selon Arnold, déterminer rapidement un village à se soumettre.

De toutes ces expéditions, seule l'expédition menée contre les Mbaza par Aiuti cite le nombre de morts, près de 60 au total ; dans les autres cas, et surtout du temps de Arnold où les opérations de guerre avaient constamment eu lieu, on ne parle que de "quelques coups de feu" qui avaient été tirés ou les "soldats avaient été contraint de faire usage de leurs armes", etc. Dans les deux opérations les plus sanglantes qui furent dirigées par Louis Royaux en 1901 et 1902 contre les Ndekere et les Vote, pas un seul mort n'a été cité, les Ndekere se réfugièrent cependant en grand nombre sur la rive droite.

En effet, à chaque expédition punitive, il y avait des morts. L'opération combinée des détachements de Yakoma et de Banzyville en février 1901 contre les Vote et les Ndekere, laissa derrière elle plusieurs morts. Les Ndekere racontèrent ceci dans leur langue ngbandi : "Zo kpi, nda Zo ko", ce qui se traduit par "un véritable massacre, ayant entrâ̂né la quasi extermination des populations"42. Cette expression, en dehors de quelque réserve qu'on peut formuler, traduisait fort bien la situation, vue l'importance de la force engagée dans cette opération.

Le silence d'Arnold et des autres agents de l'Etat pour chiffrer les victimes des "visites" des détachements de la police, trouvait vraisemblablement son explication d'une part dans le blâme dont il avait été l'objet de la part du Gouverneur, lorsque, en janvier 1901, il avait attaqué un village Mbanza du Sud-Ubangi et avait signalé la mort de 200 personnes. Par ailleurs, il fallait tenir compte de la critique de l'opinion publique après la publication du rapport de la Commission d'enquête. Il y avait lieu de taire le nombre des victimes des représailles militaires, surtout dans le territoire exploité par l'Etat Indépendant du Congo, pour sauvegarder l'image de celui-ci.

Devant l'épuisement de leur forêt et l'exigence de l'Etat, les populations du poste ont commencé à acheter leur caoutchouc sur la rive droite, dans les territoires français, où les populations semblent moins imposées ${ }^{43}$. Les habitants étaient parfois obligés de vendre tous les produits de leurs champs pour satisfaire les impositions. Il en résulta des conséquences catastrophiques pour les populations.

\section{Conséquences de l'exploitation du caoutchouc}

\section{Conséquences démographiques}

28 L'instabilité provoquée par les impositions a contribué à la régression de la population. Les nombreuses expéditions punitives ont provoqué l'exode sur la rive droite. Ces gens ne sont jamais tous rentrés. En janvier 1903, le lieutenant Arnold lui-même reconnait les faits: "La situation au poste n'est pas très bonne. Les nombreux recrutements de pagayeurs dépeuplent la rive, et font passer plusieurs villages sur la rive française". En mars, il revient sur la question de l'exode. "Pendant le mois de mars, je suis allé visiter tous les villages de la rivière en aval. Ceci pour éviter l'exode des gens de notre rive vers la rive française. Ce mouvement n'est pas encore terminé. A la rive française, les indigènes mènent une vie de paresse qui leur plaît bien. Ils ne travaillent pas ou presque pas, on n'exige d'eux aucun travail. Naturellement, cette vie sourit à nos administrés, et au premier travail qu'on exige d'eux, ils passent la frontière" ${ }^{\prime 4}$. 
29 Mais l'exploitation du caoutchouc a surtout favorisé le développement de la maladie du sommeil, cause première de l'extermination de villages entiers. Accablés par des impositions, des hommes essaient de se sauver dans la forêt, mais ils ne peuvent résiter à de dures conditions de vie loin de leurs villages et de leurs champs. La propagation de la maladie du sommeil à partir de 1905 est rendue facile dans une population se nourrissant mal, affaiblie moralement et physiquement ${ }^{45}$. Il est significatif de constater que la maladie du sommeil a surtout fait des ravages dans les régions autour du poste, dans les villages récolteurs suivants : Basa, Vote, Dondo et Ndekere à l'est ; Ngonda et Lite à l'ouest ; par contre les Mbanza à l'ouest des Lite, qui étaient moins imposés, n'étaient que très peu touchés par cette maladie.

\section{Conséquences politiques}

L'exploitation du caoutchouc a ébranlé partout l'unité des villages, soit par la mort du chef de village lors des représailles militaires, soit par sa fuite, ce qui entraînait la dispersion des habitants. Cette situation a permis à certains individus des clans cadets, voire des étrangers au village, de revendiquer le pouvoir, dans bien des cas avec la bénédiction du Blanc. Les difficultés résultant de ce changement subsistent encore de nos jours dans de nombreux groupements.

\section{Conséquences économiques}

31 Les impôts en caoutchouc, en vivres, en pagayages... ont fait disparaître les marchés. Les riverains Sango, Gbanziri, etc. sont obligés de livrer leur poisson au poste. Dans les villages de l'intérieur, les champs sont continuellement détruits par d'incessantes expéditions punitives et des occupations militaires qui en sont les corollaires. L'occupation durait parfois jusqu'à six semaines. Des villages comme Vote, Ndekere et Lite connaissaient deux à trois occupations par an. Pendant l'occupation, soldats et officiers blancs vivaient sur les villages grâce à un régime de pillage systématique : vol de poules, canards, chèvres et moutons. Bref, l'occupation était pour un village plus dévastratrice qu'une nuée de sauterelles ${ }^{46}$. Les récolteurs sont supposés se trouver en forêt vingt six jours sur trente, manquant ainsi de temps pour leur champ. En 1902, le lieutenant Arnold accorde deux mois de repos à tous les imposés pour leurs besognes (défrichement des champs, réparation des maisons...). Les travaux des champs impliquant le défrichement, l'abattage des arbres, le brûlis et le semis, les deux mois accordés pour ce travail sont insuffisants. Les femmes sont réduites à cultiver deux à trois ans de suite le même champ, ce qui entraîne la diminution de la productivité.

\section{Conséquences sociales}

La longue absence des récolteurs a pour effet de les priver de leur femmes mais surtout de les remplacer par les soldats, "des sentinelles". Ces soldats n'hésitent pas à tuer le mari qui ose faire une quelconque réclamation. La présence continuelle des soldats dans les villages et la fréquentation du poste par des femmes, soit pour livrer des vivres, soit pour faire des travaux, a pour conséquence la propagation des maladies vénériennes, notamment de la syphilis... La transmission de ces maladies par les femmes à leurs maris est le résultat de l'adultère autrefois sévèrement sanctionné par la société. 


\section{Conclusion}

\section{NOTES}

1. STENGERS, J. (1977), "Combien le Congo a-t-il coûté à la Belgique ?", Ar, roy. des Sc. Col., Bruxelles, p. 146.

2. Cfr. Bulletin Officiel, 1892, p. 307.

3. Archives Africaines, Fonds IRCB (721) 61 Concessions accordées par l'Etat Indépendant du Congo.

4. Cfr. Bulletin Officiel, mai-juin 1902, p. 151. Cfr. également J. STENGERS, op.cit., p. 152.

5. VANGROENWEGHE D., (1986), "Du sang sur les lianes. Léopold II et son Congo", Hatier, Bruxelles, p. 17.

6. Cfr. lettre du 6 octobre 1932 de Louis Royaux à Basile Tanghe, Archives des Capucins Belges, ACB, III. Ubangi. 1. Correspondances.

7. COOSEMANS, M., Royaux (Louis-Joseph) (28.11.1866-7.8.1936), dans Biographie Coloniale Belge, t. III, Bruxelles, 1952, col. 756-758.

8. ROYAUX L., (1931), Souvenirs de l'Ubangi (1892-1902) au Nord de l'Ubangi et du Mbomu. Textes recueillis et publiés par B. Tanghe, Congo, I, Bruxelles, p. 377. Cfr A. LACROIX -FIVEZ (Victor-Léon) (1855-1939) dans Biographie Coloniale Belge, t. III, Bruxelles, 1952, col. 304-307.

9. VANGROENWEGHE D., op.cit., p. 55. 
10. SALMON P., (1966), Les carnets de campagne de Georges Bricusse (6 février 1894-18 juillet 1896), CEMUBAC, Bruxelles, LXXVI, p.56. Cfr. également E. BOELAERT (1952), Ntange, dans Equatoria, II, 15, Coquilathville, p. 59.

11. ROYAUX L., art. cit., p. 378.

12. ROYAUX L., art. cit., p. 379, cfr. également P. SALMON, La révolte batetela de l'expédition du Haut-Ituri (1897). Témoignages inédits, Ac. roy. sc. o.m., Bruxelles, 1977.

13. ROYAUX L., Art. cit., pp. 382-383.

14. ROYAUX L., art. cit., p. 381.

15. Cfr. J.M. JADOT, Arnold (Rodolphe-Gaspard) (23.3.1876-26.6.1930) dans Biographie Coloniale Belge, t. III., Bruxelles, 1952, col. 19-21.

16. Cfr. lettre du 12 novembre 1901 du Gouverneur Général à A. Baert. Archives Africaines, IRCB (722) 73. Correspondances échangées entre Baert et léopold II.

17. NGBOKOTO, entrevue du 28 juin 1988 au village Yigbo (Ndekere). Sur le surnom Zebayi, cfr. Jadot Arnold..., op.cit., col. 19-21.

18. Cfr. PULIERI (Dr), AIUTI (Giovanni) (4.10.1860-16.1.1913) dans Biographie Coloniale Belge, t. III., Bruxelles, 1952, col. 8-9.

19. Cfr. A. LACROIX, Sauber (Jacques-Désiré) (27.7.1869-17.2.1921) dans Biographie Coloniale Belge, t. III., Bruxelles, 1952, col. 784-785.

20. Cfr. VAN DER LINDEN F., Dubreucq (Fernand-Louis-Joseph) (3.9.1874-15.2.1929) dans Biographie Coloniale Belge, t. II., Bruxelles, 1951, col. 302-303.

21. STENGERS J., (1989), Congo, mythes et réalités. 100 ans d'histoire, Duculot, Bruxelles, p. 97.

22. Cfr. lettre du 2 avril 1892 de Van Eetvelde au Gouverneur Général, Archives Générales du Royaume, Papiers, Van Eetvelde (35) 36 correspondances avec les Gouverneurs Généraux Ledeganck et Wahis.

23. STENGERS, J., op.cit., p. 97.

24. Archives des Capucins Belges, ACB.VI.83 (66) Rapports politiques de Banzyville.

25. VANDERVELDE E., (1911), La Belgique et le Congo, Paris, p. 50.

26. VAQUIER R., (1986), Aux temps des factoreries (1900-1950), Paris, p. 35. Cité par P. Salmon (1990), "La carrière africaine de Harry Bombeeck, agent commercial (1896-1899)", Bruxelles, pp. 10-11.

27. ...

28. AIUTI G., Cfr. Rapport cité ci-dessus.

29. DUBREUCQ, Rapport politique du mois de mars 1907. Cfr. A.C.B. VI.83 (66) Rapports politiques de Banzyville.

30. AIUTI G., Rapport spécial concernant la récolte des produits domaniaux, Banzyville, le 25 mars 1905.

31. COQUERY-VIDROVITCH C. (1972), Le Congo au temps des grandes compagnies concessionnaires (1898-1930), Paris, p. 166. Cité par P. Salmon op.cit., p. 11.

32. AIUTI G., Cfr. Rapport cité ci-dessus.

33. Cfr. A.C.B. VI.83 (66) Rapports politiques de Banzyville, 1902-1908.

34. Cfr. NGBOKUDE Selo, entrevue du 28 juin 1988 à Sokoro (Ndekere).

35. Cfr. ARNOLD R., Rapport politique du mois de mars 1902. Cfr également A.C.B. VI. 83 (66) Rapports politiques de Banzyville, 1902-1908.

36. ARNOLD, R., Cfr. Rapport cité ci-dessus.

37. ARNOLD R., Rapport politique du mois de juin.

38. AIUTI G., Rapport politique du mois de janvier 1904. Cfr. A.C.B. VI.83 (66). Rapports politiques de Banzyville.

39. AIUTI G., Rapport politique du mois de janvier 1904.

40. Le Gouverneur Général Wahis est, selon Vangroenweghe, l'instigateur de la méthode de prise d'otages. Cfr. VANGROENWEGHE, op.cit., p. 74. 
41. ARNOLD R., Rapport politique du mois d'avril 1903.

42. Cfr. NGBOKUDE Selo, entrevue du 28 juin 1988 à Sokoro (Ndekere). Cfr également R. ARNOLD, Rapport politique du mois de mars 1902.

43. Cfr. lettre du 7 mars 1906 du Gouverneur Général à A. Baert. Cfr. IRCB (722) 73. Correspondances échangées entre Baert et Léopold II.

44. ARNOLD R., Rapport politique du mois de mars 1902.

45. Cfr. Rapport de la Commission d'enquête dans Bulletin Officiel, 1905, pp. 236-237. Cfr également VANDERVELDE E., op.cit., p. 104.

46. VANDERVELDE E., op.cit., p. 98.

47. Cfr lettre du 6 octobre 1933 de L. Royaux à Tanghe. Arch. citées ci-dessus. Cfr également ROYAUX, L., Souvenirs..., art.cit., p. 381.

\section{RÉSUMÉS}

According to Léopold II, the profitability of the Congro (Zaïre) was to be assured through the exploitation of rubber. However, the harvest of this product was carried out with violence as illustrated in the example of the Banzyville territory (Mobayi-Mbongo), far from the eyes of protestant missionaries. This study, based on an unpublished document, shows the working conditions of the population left both in the hands of and to the whims of officers of the "Etat Indépendant du Congo". Between 1902 and 1908, the territory produced 156,646 kg of rubber. This production was obtained at the cost of enormous brutality inflicted on the population. Numerous revolts were cruelly put down. The principle figures in these atrocities were the lieutenants Louis Royaux and Rodolphe Arnold as well as captain Ciovanni Aiuti. None of these officers was taken to court. The exploitation of rubber has left the population of Banzyville still nursing these painful memories.

\section{AUTEUR}

\section{TE MOBUSA NGBWAPKWA}

Université de Kinshasa - Kinshasa - République du Zaïre 